\title{
Deutsche Politikwissenschaft: Nationale Tradition und internationale Aufmerksamkeit
}

\author{
A. J. J. Ton Nijhuis
}

Online publiziert: 9. März 2017

(C) Der/die Autor(en) 2017. Dieser Artikel ist eine Open-Access-Publikation.

Die deutsche Politikwissenschaft besitzt traditionell einige Besonderheiten, die sie im internationalen Feld deutlich von ihren Partnerdisziplinen in anderen Ländern unterscheidet. Einer der Hauptgründe hierfür liegt in der herausgehobenen gesellschaftlichen Stellung, die das Fach nach dem Zweiten Weltkrieg in Deutschland einnahm, da ihm eine wichtige Rolle bei der Entwicklung des demokratischen Rechtsstaats und einer demokratischen politischen Kultur zugeschrieben wurde. Diese Sonderrolle als Demokratiewissenschaft entsprach durchaus auch dem disziplinären Selbstverständnis der Fachvertreter, die sich auf allen Ebenen aktiv an öffentlichen Debatten beteiligten. Wenngleich man meinen möchte, dass sich dies inzwischen geändert hat und sich die deutsche Politikwissenschaft - nach einer starken Ideologisierung in den 1970er und 80er-Jahren - in eine ,ganz normale“ international anschlussfähige Disziplin transformiert hat, sind bis heute deutliche Spuren dieser ursprünglichen Ausrichtung sichtbar. Nach wie vor sehen viele deutsche Politikwissenschaftler ihre Aufgabe (auch) darin, einen Beitrag zur Weiterentwicklung von Demokratie und Rechtsstaat zu leisten.

Die gesellschaftliche Sonderrolle der deutschen Politikwissenschaft stand in einem mitunter direkten Zusammenhang mit weiteren disziplinären Eigenheiten. Da ist zum einen ihre starke historische Orientierung, die sich nicht zuletzt aus einer verständlich hohen Sensibilität gegenüber der Notwendigkeit einer Aufarbeitung der deutschen Geschichte erklärt. Im Wechselverhältnis hierzu steht zudem eine - verglichen mit anderen Ländern - ungewöhnliche Vorliebe für fortschrittsorientierte Themen und Konzepte, welche Perspektiven auf eine bessere Welt eröffnen. Anschauliche Beispiele hierfür geben zum Beispiel im Bereich der Internationalen Be-

Prof. Dr. A. J. J. T. Nijhuis ( $₫)$

Universiteit van Amsterdam, Kloveniersburgwal 48, 1012CX Amsterdam, Niederlande

E-Mail: A.J.J.Nijhuis@uva.nl 
ziehungen die kontrastive Analyse der Bedeutung und Ausprägung von Zivilmacht oder auch die einschlägigen Forschungen zur Global Governance.

Dieser Sonderweg, den die deutsche Politikwissenschaft nach dem Zweiten Weltkrieg einschlug, stieß indes durchaus auf internationales Interesse. Dabei lag die hohe Attraktivität, welche das Fach speziell in den Niederlanden besaß, vor allem in seinem hohen Theoriepotenzial begründet. Gleich ob es sich um Staatstheorien, politische Philosophie, Ideengeschichte oder Soziologie handelte, stellte die deutsche Politikwissenschaft für die theoretisch interessierten niederländischen Kollegen lange Zeit eine überaus reiche Inspirationsquelle dar. Dies galt sogar für die 1970er und 80er-Jahre, als sich ein nicht unbeachtlicher Teil der politikwissenschaftlichen Forschung in Deutschland stark ideologisch einfärbte. So stießen beispielsweise die Frankfurter Schule und die marxistischen Theorien in den Niederlanden auf breite Zustimmung.

Inzwischen sind die beschriebenen Besonderheiten der deutschen Politikwissenschaft allerdings deutlich verblasst. Obwohl sich diese Entwicklung nicht monokausal erklären lässt, ist der Hauptgrund hierfür sicherlich im Wunsch nach einer stärkeren internationalen Ausrichtung und Anbindung zu suchen, der mit der zunehmenden Notwendigkeit korrespondiert, politikwissenschaftliche Forschung über zweite und dritte Geldströme zu finanzieren. Auf der Basis dieser Internationalisierungstendenz hat sich das Fach grundlegend geändert und vor allem eine empirische Wende durchlebt. Die Zeit großer Theoriedebatten ist endgültig vorbei. Auch die historische Ausrichtung spielt eine deutlich geringere Rolle als früher.

Diese - letztlich auf der stärkeren Orientierung am angelsächsischen Mainstream fußenden - „Normalisierung“ hat allerdings nicht nur die Anschlussfähigkeit der Disziplin erhöht, sondern paradoxerweise auch dazu geführt, dass die deutsche Politikwissenschaft für das Ausland im Allgemeinen und die Niederlande im Besonderen deutlich an Attraktivität verloren hat. Gerade ihre Eigenheiten waren es ja, vor allem ihre starke Bindung an Theorien und Konzepte, welche der internationalen Forschung über viele Jahrzehnte hinweg wichtige Impulse lieferte und sie auf diesem Weg auch nachhaltig beeinflusst hat. Inzwischen hat sich dies geändert: Die deutschen Theorie-Exporteure kommen heutzutage eher aus der Soziologie - wie beispielsweise Jürgen Habermas oder Ulrich Beck mit seinem Konzept der Risikogesellschaft. Von der neuen, eher empirisch orientierten deutschen Politikwissenschaft sind auf theoretischem Gebiet kaum noch innovative Ansätze zu erwarten.

Damit aber befindet sich die deutsche Politikwissenschaft aktuell in einem Dilemma: Um international mehr Einfluss auszuüben und mehr Aufmerksamkeit zu bekommen, müsste sie sich eigentlich vom anglo-amerikanischen Mainstream etwas distanzieren und sich stattdessen wieder mehr auf ihre alten Stärken konzentrieren. Eine solche mögliche Theoriewende würde allerdings zweifellos auch Probleme generieren, insofern empirische, projektgebundene Forschungsarbeiten eher Aussicht auf Erfolg im Rennen um die begehrten Finanzierungen bieten als theoretische Analysen. Hier müssten Konzepte gefunden werden, die beide Aspekte sinnvoll miteinander kombinieren.

Ein weiteres Problem besteht darin, dass die Steigerung der internationalen Anschlussfähigkeit in der Regel auf Kosten der nationalen Relevanz geht. Dabei ist das Risiko groß, dass die zur Profilierung eines Wissenschaftlers heute unabdingbaren 
Publikationen in den einschlägigen angelsächsischen Peer-Reviewed-Zeitschriften langfristig einen Rückgang der Erforschung spezifisch deutscher Themen und Bereiche provoziert. Ein anschauliches Beispiel hierfür ist die Erforschung der politischen Institutionen und Prozesse auf Ebene der Bundesländer, die eindeutig nicht dazu geeignet erscheint, internationale Wahrnehmung und Anerkennung zu erhalten. Hier droht die Entstehung einer Politikwissenschaft erster und zweiter Klasse. Darüber hinaus entsteht durch die Internationalisierung aber auch eine Kluft zwischen der akademischen Forschung und der politischen Praxis, das heißt die immer stärkere Verwissenschaftlichung im internationalen Kontext führt zu einem Verlust an gesellschaftlicher Relevanz. Dieses Problem findet seine Entsprechung auch in der universitären Lehre. Will man die Studierenden der Politikwissenschaft auf eine internationale akademische Karriere als Wissenschaftler vorbereiten oder zu Experten und Beratern in der deutschen Politik, Verwaltung oder Öffentlichkeitsarbeit machen?

Hier zeigt sich - und diese Feststellung lässt sich in gewisser Weise sicherlich auch auf andere Disziplinen und Länder übertragen -, dass der Preis, den wir für die fortschreitende internationale Verwissenschaftlichung zu zahlen haben, der Verlust an nationaler bzw. regionaler Relevanz und Kompetenz ist - und umgekehrt. So bedarf es aus dieser Perspektive zur Erhöhung der gesellschaftlichen Bedeutung und Einflussnahme politikwissenschaftlicher Forschung mehr Mut zur Provinzialisierung. Welcher Weg hier einzuschlagen ist, kann natürlich jeder Wissenschaftler seinen individuellen Vorlieben und Neigungen entsprechend entscheiden, für die Disziplin als Ganzes betrachtet stellt diese Wahl indes ein existentielles Dilemma dar.

Open access funding provided by University of Amsterdam.

Open Access Dieser Artikel wird unter der Creative Commons Namensnennung 4.0 International Lizenz (http://creativecommons.org/licenses/by/4.0/deed.de) veröffentlicht, welche die Nutzung, Vervielfältigung, Bearbeitung, Verbreitung und Wiedergabe in jeglichem Medium und Format erlaubt, sofern Sie den/die ursprünglichen Autor(en) und die Quelle ordnungsgemäß nennen, einen Link zur Creative Commons Lizenz beifügen und angeben, ob Änderungen vorgenommen wurden. 